\title{
La néphropathie non diabétique chez les patients diabétiques de type 2 à l'hôpital militaire Mohammed V de Rabat (Maroc)
}

Y. Zajjari, ${ }^{7}$ M. Benyahia, ${ }^{1}$ D. Montasser Ibrahim, ${ }^{1}$ J. Kassouati, ${ }^{2}$ O. Maoujoud, ${ }^{1}$ F. El Guendouz ${ }^{3}$ et Z. Oualim ${ }^{7}$

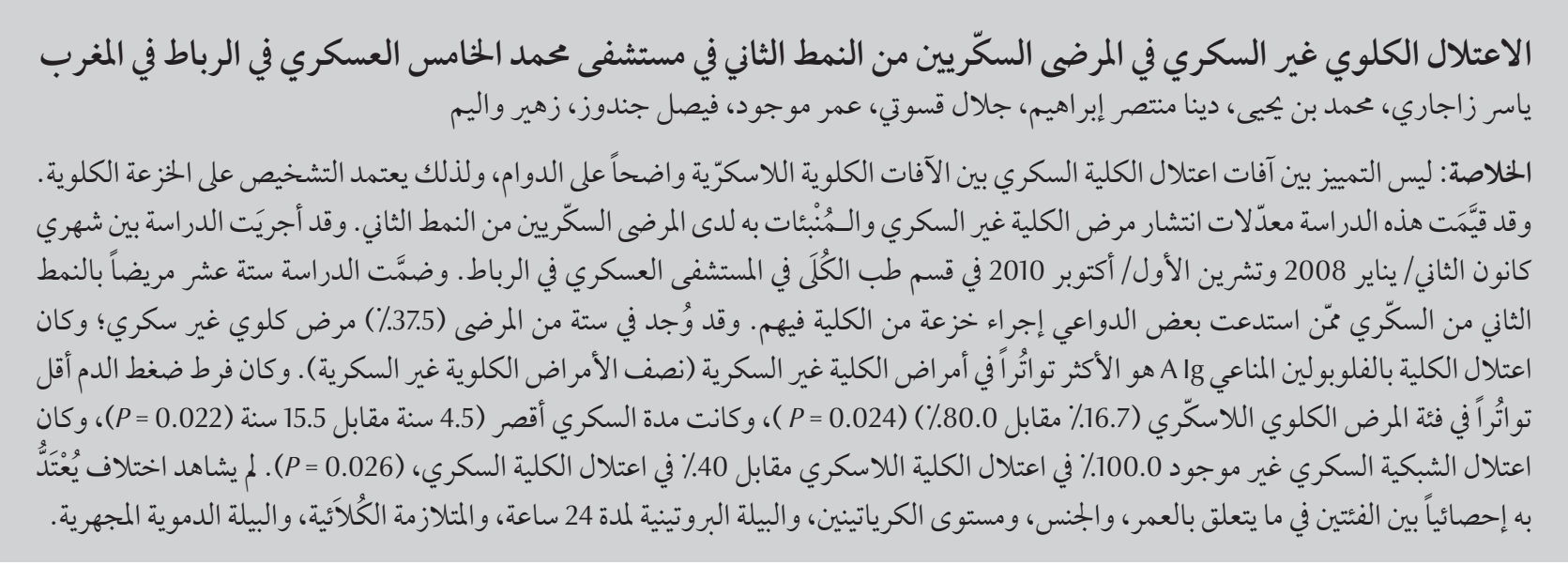

RÉSUMÉ La distinction entre les lésions de néphropathie diabétique et les lésions de néphropathie non diabétique n'est pas toujours évidente; elle est basée souvent sur la ponction-biopsie rénale. Cette étude a évalué la prévalence et les facteurs prédictifs de la néphropathie non diabétique chez le diabétique de type 2. L'étude, réalisée entre janvier 2008 et octobre 2010 au service de néphrologie de l'hôpital militaire de Rabat, incluait 16 diabétiques de type 2 ayant bénéficié d'une ponction-biopsie rénale. La néphropathie non diabétique a été observée chez 6 patients $(37,5 \%$; la néphropathie à IgA était la plus fréquente (50\% des néphropathies non diabétiques). L'hypertension artérielle était significativement moins fréquente dans le groupe de la néphropathie non diabétique que dans le groupe de la néphropathie diabétique $(16,7 \%$ contre $80,0 \% ; p=0,024)$, la durée du diabète était plus courte $(4,5$ contre 15,5 ans ; $p=0,022)$ et la rétinopathie diabétique absente $(100 \%$ contre 40 $\% ; p=0,026)$. II n'y avait pas de différence statistiquement significative entre les deux groupes en ce qui concerne l'âge, le sexe, la créatininémie, la protéinurie des 24 h, le syndrome néphrotique et l'hématurie microscopique.

\begin{abstract}
Non-diabetic renal disease in type II diabetes mellitus patients in Mohammed V military hospital, Rabat, Morocco
ABSTRACT The distinction between diabetic nephropathy lesions and non-diabetic renal lesions is not always obvious and is often based on renal biopsy. This study evaluated the prevalence and predictors of nondiabetic renal disease in people with type 2 diabetes. The study was conducted between January 2008 and October 2010 in the nephrology department of the military hospital in Rabat. The study included 16 patients with type 2 diabetes in whom renal biopsy was indicated. Non-diabetic renal disease was found in 6 of the patients (37.5\%); IgA nephropathy was the most frequent non-diabetic renal disease (half of non-diabetic renal diseases). Hypertension was significantly less frequent in the non-diabetic renal disease group than the diabetic nephropathy group (16.7\% versus $80.0 \%, P=0024)$, duration of diabetes was a shorter (4.5 versus 15.5 years, $P=$ $0.022)$ and diabetic retinopathy was absent (100\% versus $40 \%, P=0.026)$. There were no statistically significant differences between the 2 groups in relation to age, sex, creatinine level, 24-hour proteinuria, nephrotic syndrome and microscopic haematuria.
\end{abstract}

'Service de Néphrologie, Dialyse et Transplantation rénale ; ${ }^{2}$ Service d'Endocrinologie, Hôpital militaire d'instruction Mohammed V, Rabat (Maroc). ${ }^{3}$ Laboratoire de Biostatistique, de Recherche Clinique et d'Épidémiologie, Faculté de Médecine et de Pharmacie, Rabat (Maroc).

(Correspondance à adresserà Y. Zajjari :cap.yas@hotmail.com).

Reçu : 18/12/11; accepté : 26/01/12 


\section{Introduction}

La néphropathie diabétique (ND) est la première cause d'insuffisance rénale chronique terminale dans le monde [1] ; elle complique le diabète dans $40 \%$ des cas avec une ancienneté de diabète de plus de 20 ans [2,3]. Généralement, le diagnostic de la ND est aisé si le diabète est ancien, en présence de complications dégénératives et lorsque l'évolution est marquée par une protéinurie précédant l'insuffisance rénale.

Si cette démarche est validée chez le diabétique de type 1, elle est discutée chez le diabétique de type 2 [4]. L'absence de neuropathie et de rétinopathie diabétique (RD) en présence de signes de néphropathie doit faire suspecter une néphropathie non diabétique (NND) [5], de même qu'une détérioration rapide de la fonction rénale ainsi que la présence d'une hématurie macro- ou microscopique [6]. La ponction-biopsie rénale (PBR) n'est habituellement indiquée chez le diabétique de type 2 que si une NND est suspectée. Le but de l'étude était d'évaluer la prévalence de la NND et les facteurs associés chez le diabétique de type 2 .

\section{Méthodes}

Il s'agit d'une étude rétrospective réalisée entre janvier 2008 et octobre 2010 au service de néphrologie de l'hôpital militaire de Rabat, incluant les PBR effectuées chez les patients ayant un diabète de type 2 , selon la définition de l'Organisation mondiale de la Santé, chez lesquels le diagnostic de NND était suspecté.

La PBR était réalisée par voie percutanée après consentement éclairé des patients, en utilisant un dispositif semi-automatique armé par une aiguille à usage unique (16 Gauge). L'étude histologique était réalisée par microscopie optique et par immunofluorescence aux antisérums IgG, IgA, IgM, C3, C4, C1q et fibrinogène, et dans certains cas aux antisérums des chaînes légères Kappa et Lambda par le même anatomopathologiste. Nous avons recueilli des paramètres démographiques, cliniques et biologiques.

L'étude statistique a été réalisée par un logiciel SPSS 13.0. Les variables quantitatives étaient exprimées en médianes et les variables qualitatives en pourcentages. La comparaison des variables quantitatives était réalisée par le test non paramétrique de MannWhitney et la comparaison des variables qualitatives était réalisée par le test exact de Fischer. Une valeur de $p<0,05$ était retenue comme statistiquement significative.

\section{Résultats}

Durant la période d'étude, 16 PBR ont été réalisées chez 15 patients par voie percutanée et dans un cas sur pièce de néphrectomie pour tumeur rénale ; $81,3 \%$ étaient des patients de sexe masculin ayant un diabète de type 2, évoluant depuis plus de 6,5 ans chez la moitié. La médiane d'âge était de 60 ans (extrêmes 47-79 ans). Neuf patients (56,3\%) étaient hypertendus, 10 patients $(62,5 \%)$ n'avaient pas de

\begin{tabular}{lc}
\hline Tableau 1 Paramètres démographiques, cliniques et biologiques des patients \\
\hline Variable & Valeur \\
Âge (ans) [médiane (quartile)] & $60(47-79)$ \\
Sexe & $13(81,3)$ \\
Masculin [Nbre (\%)] & $3(18,7)$ \\
Féminin [Nbre (\%)] & $9(56,3)$ \\
Hypertension artérielle [Nbre (\%)] & $10(62,5)$ \\
Absence de rétinopathie diabétique [Nbre (\%)] & $6,5(1-39)$ \\
Ancienneté du diabète (ans) [médiane (quartile)] & $45,25(7-112)$ \\
Créatininémie (mg/L) [médiane (quartile)] & $11(68,8)$ \\
Syndrome néphrotique [Nbre (\%)] & $4,75(0,7-13)$ \\
Protéinurie (g/j) [médiane (quartile)] & $10(62,5)$ \\
Hématurie [Nbre (\%)]
\end{tabular}

$\mathrm{RD}$. La médiane de la protéinurie de 24 heures était de $4,75 \mathrm{~g} / \mathrm{j}$ (extrêmes $0,7-13 \mathrm{~g} / \mathrm{j})$. Onze patients $(68,8 \%)$ étaient néphrotiques et $10(62,5 \%)$ avaient une hématurie microscopique. La médiane de créatininémie était de $45 \mathrm{mg} / \mathrm{L}$ (Tableau 1).

Les indications de la PBR étaient un syndrome néphrotique associé à un diabète récent (durée de moins de cinq ans) chez quatre patients, une insuffisance rénale rapidement progressive associée à une hématurie microscopique chez trois patients, une insuffisance rénale aiguë associée à des signes extrarénaux (cutanés, digestifs et articulaires) chez deux patients, une insuffisance rénale associée à des anomalies biologiques chez six patients (quatre cas de dysglobulinémies, un cas d'hépatite virale chronique $\mathrm{B}$, un cas d'hypocomplémentémie C3), et une insuffisance rénale associée à un oncocytome rénal chez un seul patient.

Sur les 16 patients diabétiques biopsiés, six patients présentaient des lésions de NND. Il s'agissait de trois cas de néphropathie à IgA (deux cas de purpurarhumatoïdeetun cas de maladie de Berger), d'un cas de néphropathie à lésions glomérulaires minimes (LGM), d'un cas de glomérulopathie extramembraneuse (GEM) et d'un cas de tubulopathie myélomateuse. 
L'ancienneté du diabète était plus marquée dans le groupe ND en comparaison avec le groupe NND (15,5 contre 4,5 ans; $p=0,022)$. L'hypertension artérielle était moins fréquente dans le groupe NND $(16,7 \%$ contre $80,0 \% ; p=$ 0,024).La RD était absente chez tous les patients du groupe NND et chez $40 \%$ des patients du groupe ND $(p=0,026)$. Il n'y avait pas de différence significative entre les deux groupes concernant l'âge, le sexe, la créatininémie, la protéinurie de 24 heures, le syndrome néphrotique et l'hématurie microscopique (Tableau 2).

\section{Discussion}

Dans ce travail, la PBR était réalisée chez 16 patients diabétiques de type 2, dans un contexte de suspicion de NND expliquant en partie le faible échantillon de notre série. Les critères de suspicion de NND chez le diabétique de type 1 sont: l'hématurie microscopique, l'absence de $\mathrm{RD}$, l'insuffisance rénale d'évolution rapide ou la présence d'anomalies immunologiques. Cependant ces critères ne sont pas validés chez le diabétique de type 2 [3].

Dans les séries de biopsies rénales chez le diabétique de type 2 , la prévalence de la NND varie de 10 à
$85 \%[4,7-10]$; cette différence dépend des critères d'indication de la PBR et de la population étudiée [11]. Dans notre étude, la prévalence de la NND était de $37,5 \%$. Les néphropathies à IgA étaient les étiologies les plus fréquentes des NND (50\% des cas). Dans la série de Zhou et al, la néphropathie à IgA (34\%) et les GEM (22\%) étaient les étiologies les plus fréquentes de NND [12].

La ND est une complication chronique du diabète; elle se manifeste cliniquement 5 à 10 ans après la découverte du diabète. Ainsi, une courte durée d'évolution du diabète en présence d'anomalies rénales oriente vers une NND [12]. Dans la série de Choi, les NND étaient associées à une courte durée du diabète [4]. Dans notre série, le diabète était d’ancienneté plus courte dans le groupe NND avec une médiane de 4,5 ans contre 15,5 ans dans le groupe $\mathrm{ND}(p=0,022)$.

La RD est une complication microvasculaire du diabète de pathogénie similaire à la ND [12]. Chez le diabétique de type 1, elles coexistent habituellement selon plusieurs auteurs $[13,14]$. Selon Parving, tous les diabétiques de type 2 ayant une protéinurie avec une $\mathrm{RD}$ ont une $\mathrm{ND}$; cependant, en l'absence de $\mathrm{RD}$ avec une protéinurie, $50 \%$ des patients avaient une ND [15]. Dansla série de Zhou etal., $90 \%$ des diabétiques de type 2 avec RD avaient une ND et $76 \%$ des diabétiques de type 2 sans $R D$ avaient une NND, ce qui suggère que la présence de la $\mathrm{RD}$ est un marqueur de ND et que son absence est un facteur prédictif de NND [12]. Dans notre série, tous les patients ayant une RD avaient une ND et $60 \%$ des patients sans $\mathrm{RD}$ avaient une NND.

L'hypertension artérielle est une expression fréquente des néphropathies; cependant, elle est plus fréquente chez le diabétique. Cela s'explique par la rétention hydrosodée, l'activation du système rénine angiotensine, la stimulation du système sympathique et le dysfonctionnement endothélial plus fréquent chez cette population [12]. Dans la série de Zhou et al., l'hypertension artérielle était plus fréquente et plus sévère dans le groupe ND [12] ; dans notre série, le groupe NND avait moins d'hypertension artérielle (16,7\% contre $80 \% ; p=0024)$.

L’hématurie est une manifestation inhabituelle de la ND. Elle est l'expression de plusieurs NND, notamment les néphropathies à IgA. Dans l'étude de Wong, l'association de la protéinurie ou de l'hématurie à l'absence de RD orientait vers une NND avec une valeur prédictive positive de $94 \%$ [16]. Dans notre série, l'hématurie était présente chez $83,3 \%$ des patients du groupe NND et $50 \%$

\begin{tabular}{lccc}
\hline Tableau 2 Comparaison du groupe ND et groupe NND & & & \\
\hline Caractéristiques & Groupe ND & Groupe NND & $p$ \\
Âge (ans) [Médianes (quartiles)] & $60(56,25 ; 63)$ & $56,5(50,75 ; 62)$ & 0,229 \\
Sexe masculin [Nbre (\%)] & $8(80)$ & $5(83,8)$ & 0,696 \\
Hypertension artérielle [Nbre (\%)] & $2(80)$ & $5(16,7)$ & $0,024^{*}$ \\
$\begin{array}{l}\text { Absence de rétinopathie diabétique } \\
\text { [Nbre (\%)] }\end{array}$ & $4(40)$ & $6(100,0)$ & $0,026^{*}$ \\
Ancienneté du diabète (ans) [Médianes & & $0,022^{*}$ \\
(quartiles)] & $15,5(5,75 ; 20,25)$ & $4,5(1 ; 6,25)$ & 0,142 \\
Créatininémie (mg/L) & & & 0,828 \\
[Médianes (quartiles)] & $56,5(32,25 ; 83,25)$ & $5,1(2,87 ; 11,2)$ & 0,215 \\
Protéinurie (g/j) [Médianes (quartiles)] & $4,65(3,42 ; 8,12)$ & $5(83,3)$ & \\
Hématurie [Nbre (\%)] & $5(50)$ & & \\
\hline
\end{tabular}

*Significatif à $p<0,05$.

$N D$ : néphropathie diabétique; NND : néphropathie non diabétique. 
des patients du groupe ND. Cependant, il n'y avait pas de différence statistiquement significative ( $p=$ 0,215). Cela peut être expliqué en partie par le faible échantillon de notre série en l'absence d'indication systématique de PBR chez le diabétique de type 2 .

\section{Conclusion}

Bien que de nombreux indicateurs puissent orienter vers le diagnostic de NND chez le diabétique de type 2 , l'étude histologique du parenchyme rénal reste le moyen diagnostique le plus sûr pour la prise en charge et le pronostic de ces patients. Toutefois, le risque de complications hémorragiques encourus lors de la PBR doit limiter ces indications systématiques. L'échantillon faible de notre série rend nécessaire des études plus larges.

\section{Références}

1. Held PJ et al. The United States renal data system's 1990 annual data report: an introduction. American Journal of Kidney Disease, 1990 16(Suppl. 2):1-106.

2. Olsen S. Identification of non-diabetic glomerular disease in renal biopsies from diabetics: a dilemma. Nephrology, Dialysis, Transplantation, 1999, 14:1846-1849.

3. Serra A et al. Is there a need for changes in renal biopsy criteria in proteinuria in type 2 diabetes. Diabetes Research and Clinical Practice, 2002, 58:149-153.

4. Lee EY, Chung $\mathrm{CH}$, Choi SO. Non-diabetic renal disease in patients with non-insulin dependent diabetes mellitus. Yonsei Medical Journal, 1999, 40:321-326.

5. Oslerby R et al. Glomerular structure and function in diabetes mellitus. Diabetes, 1990, 39:1057-1060.

6. Parving HH, Osterby R, Ritz E. Diabetic nephropathy. In: Brenner BM, ed. Brenner \& Rector's The Kidney. Philadelphia, WB Saunders, 2000:1731-1760.

7. Olsen S, Mogensen CE. How often is NIDDM complicated with non-diabetic renal disease? An analysis of renal biopsies and the literature. Diabetologia, 1996, 39:1638-1645.

8. Lee EY, Chung $\mathrm{CH}$, Choi SO. Non-diabetic renal disease in patients with non-insulin dependent diabetes mellitus. Yonsei Medical Journal, 1999, 40(4):321-326.

9. Nzerue $\mathrm{CM}$ et al. Prevalence of non diabetic renal disease among African-American patients with type II diabetes mel- litus. Scandinavian Journal of Urology \& Nephrology, 2000, 34:331-335.

10. Prakash $\mathrm{J}$ et al. Non-diabetic renal disease in patients with type 2 diabetes mellitus. Journal of the Association of Physicians of India, 2001, 49:415-420.

11. Bertani T et al. Superimposed nephritis: A separate entity among glomerular diseases? American Journal of Kidney Disease, 1986, 7:205-212.

12. Jianhui Zhou et al. A differential diagnostic model of diabetic nephropathy and non-diabetic renal diseases. Nephrology, Dialysis, Transplantation, 2008, 23:1940-1945.

13. Hovind $\mathrm{P}$ et al. Predictors for the development of microalbuminuria and macroalbuminuria in patients with type 1 diabetes: Inception cohort study. British Medical Journal, 2004, 328:1105.

14. Perkins BA et al. Regression of microalbuminuria in type 1 diabetes. New England Journal of Medicine, 2003, 348:22852293.

15. Parving $\mathrm{HH}$ et al. Prevalence and causes of albuminuria in non-insulin-dependent diabetic patients. Kidney International, 1992, 41:758-762.

16. Wong TY et al. Renal outcome in type 2 diabetic patients with or without coexisting non diabetic nephropathies. Diabetes Care, 2002, 25(5):900-905. 